\title{
Reductive Monoalkylation of Aromatic and Aliphatic Nitro Compounds and the Corresponding Amines with Nitriles
}

Ruel Nacario, Shailaja Kotakonda, David M. Fouchard, L. M. Viranga Tillekeratne, and Richard A. Hudson

\section{General Methods:}

Reagents were purchased from Aldrich and Fischer and used without further purification. Reactions were carried out in oven-dried glassware. Unless stated otherwise, all reactions were carried out at room temperature and pressure and were monitored initially by TLC on pre-coated silica gel IB-F (4463-02 Baker-Flex, J.T. Baker), by NMR and by GC-MS. TLC separations were detected under UV light or by charring after spraying with ninhydrin solution. Column chromatography was performed on 100-200 mesh silica gel (Fisher Scientific). NMR spectra were recorded on 400 or $600 \mathrm{MHz}$ Varian NMR spectrometers. ${ }^{1} \mathrm{H}$ NMR chemical shifts were referenced to tetramethylsilane (TMS) (0.0, $\mathrm{CDCl}_{3}$ ) while ${ }^{13} \mathrm{C}$ NMR chemical shifts were referenced to $\mathrm{CDCl}_{3}\left(77.0, \mathrm{CDCl}_{3}\right)$. Coupling constants $(J)$ were expressed in Hertz $(\mathrm{Hz})$. GCMS-EI was performed on a Hewlett Packard G1800B GCD Plus Gas Chromatograph equipped with Electron Ionization Detector and a $12 \mathrm{~m} \times 0.2 \mathrm{~mm}$ column. Helium was used as the carrier gas with a constant flow of $0.5 \mathrm{~mL} / \mathrm{min}$. The oven temperature was held at $45^{\circ} \mathrm{C}$ for $4 \mathrm{~min}$ then increased at $20{ }^{\circ} \mathrm{C} / \mathrm{min}$ to $280{ }^{\circ} \mathrm{C}$ with a final holding time of $5.0 \mathrm{~min}$. Yields reported in Tables 1-4 are based on weight of isolated products; product ratios are based on GC-MS analysis of the isolated products. 
General Procedure A: A mixture of starting material (100 mg) and $5 \%$ palladium on carbon $\left(0.039 \mathrm{~g}, 1.81 \times 10^{-5} \mathrm{~mol}\right)$ in $80 \%$ acetonitrile-water $(5 \mathrm{~mL})$ was stirred at room temperature. Ammonium formate $\left(0.60 \mathrm{~g}, 6.4 \times 10^{-3} \mathrm{~mol}\right)$ was added in small portions. The reaction was monitored by TLC for the disappearance of the spot (lowest $\mathrm{R}_{\mathrm{f}}$ ) corresponding to the primary amine. The reaction mixture was then filtered, washed successively with acetonitrile and dichlomethane and the filtrate evaporated in vacuo. The residue was dissolved in $\mathrm{CH}_{2} \mathrm{Cl}_{2}$ and filtered. The filtrate was then evaporated in vacuo to give a product.

N-Ethyl-4-methoxyaniline: Starting from 4-nitroanisole $\left(0.10 \mathrm{~g}, 6.5 \times 10^{-4} \mathrm{~mol}\right)$ using general procedure A gave a dark-brown liquid $(0.09 \mathrm{~g}, 93 \%)$. $R_{\mathrm{f}} 0.43$ (10:1 hexanes:ethyl acetate) ${ }^{1} \mathrm{H}$ NMR $\left(600 \mathrm{MHz}, \mathrm{CDCl}_{3}, \delta_{\mathrm{H}}\right) 1.23(\mathrm{t}, J=7.2 \mathrm{~Hz}, 3 \mathrm{H}), 3.10(\mathrm{q}, J=7.2 \mathrm{~Hz}$, 2H), $3.74(\mathrm{~s}, 3 \mathrm{H}), 6.58(\mathrm{~d}, J=9 \mathrm{~Hz}, 2 \mathrm{H}), 6.78(\mathrm{~d}, J=9 \mathrm{~Hz}, 2 \mathrm{H}) ;{ }^{13} \mathrm{C} \mathrm{NMR}(400 \mathrm{MHz}$, $\left.\mathrm{CDCl}_{3}, \delta_{\mathrm{C}}\right) 15.2,39.7,56.1,114.3,115.1,143.0,152.3$; GCMS (EI) $m / z 151$.

N-Ethyl aniline: Starting from nitrobenzene $\left(0.10 \mathrm{~g}, 8.1 \times 10^{-4} \mathrm{~mol}\right)$ using general procedure A gave a light orange liquid $(0.08 \mathrm{~g}, 85 \%) . R_{\mathrm{f}} 0.42$ (10:1 hexanes:ethyl acetate); ${ }^{1} \mathrm{H}$ NMR (600 MHz, $\left.\mathrm{CDCl}_{3}, \delta_{\mathrm{H}}\right) 1.27(\mathrm{t}, J=7.2 \mathrm{~Hz}, 3 \mathrm{H}), 3.17$ (q, $J=7.2 \mathrm{~Hz}$, 2H), 3.63 (br. s, 1H), 6.63 (m, 2H), $6.72(\mathrm{~m}, 1 \mathrm{H}), 7.20$ (m, 2H); ${ }^{13} \mathrm{C}$ NMR (400 MHz, $\left.\mathrm{CDCl}_{3}, \delta_{\mathrm{C}}\right) 15.2,38.7,113.1,117.5,129.5,148.7$; GCMS (EI) $\mathrm{m} / z 121$. 
N-Ethyl-4-methylaniline: Starting from 4-nitrotoluene ( $\left.0.1 \mathrm{~g}, 7.3 \times 10^{-4} \mathrm{~mol}\right)$, and ammonium formate $\left(\mathrm{NH}_{4} \mathrm{HCO}_{2}, 0.80 \mathrm{~g}, 1.3 \times 10^{-3} \mathrm{~mol}\right)$ but otherwise using general procedure A gave an orange-brown liquid $(0.0747 \mathrm{~g}, 75.8 \%)$. $R_{\mathrm{f}} 0.43$ (10:1 hexanes:ethyl acetate); ${ }^{1} \mathrm{H} \mathrm{NMR}\left(600 \mathrm{MHz}, \mathrm{CDCl}_{3}, \delta_{\mathrm{H}}\right) 1.23(\mathrm{t}, J=7.2 \mathrm{~Hz}, 3 \mathrm{H}), 2.23(\mathrm{~s}, 3 \mathrm{H}), 3.13$ (q, $J$ $=7.2 \mathrm{~Hz}, 2 \mathrm{H}), 6.54(\mathrm{~d}, J=8.4 \mathrm{~Hz}, 2 \mathrm{H}), 6.78(\mathrm{~d}, J=9 \mathrm{~Hz}, 2 \mathrm{H}) ;{ }^{13} \mathrm{C}$ NMR $(400 \mathrm{MHz}$, $\left.\mathrm{CDCl}_{3}, \delta_{\mathrm{C}}\right) 15.3,20.7,39.1,113.3,126.7 ; 130.1,146.6$; GCMS (EI) $m / z 135$.

General Procedure B: Starting material $(100 \mathrm{mg})$ was dissolved in acetonitrile $(4 \mathrm{~mL})$ and stirred at room temperature. $5 \% \mathrm{Pd} / \mathrm{C}\left(0.039 \mathrm{~g}, 1.8 \times 10^{-5} \mathrm{~mol}\right)$ and $\mathrm{NH}_{4} \mathrm{HCO}_{2}(0.60$ g, $\left.9.5 \times 10^{-3} \mathrm{~mol}\right)$ dissolved in $\mathrm{H}_{2} \mathrm{O}(1.0 \mathrm{~mL})$ was then added to the reaction mixture while stirring. The reaction was monitored by TLC for the disappearance of the spot (lowest $\mathrm{R}_{\mathrm{f}}$ ) corresponding to the primary amine intermediate. The reaction mixture was then filtered, washed successively with methanol and $\mathrm{CH}_{2} \mathrm{Cl}_{2}$ and the filtrate evaporated in vacuo. The residue was dissolved in $\mathrm{CH}_{2} \mathrm{Cl}_{2}$ and the organic layer was separated, dried in $\mathrm{Na}_{2} \mathrm{SO}_{4}$ and filtered. The filtrate was then evaporated in vacuo to give the product.

N-Ethyl aniline: Starting from aniline $\left(0.10 \mathrm{~g}, 1.07 \times 10^{-3} \mathrm{~mol}\right)$ using general procedure B gave a light yellow liquid $(0.11689 .5 \%) . R_{\mathrm{f}} 0.46\left(10: 1\right.$ hexanes:ethyl acetate); ${ }^{1} \mathrm{H}$ NMR (600 MHz, $\left.\mathrm{CDCl}_{3}, \delta_{\mathrm{H}}\right) 1.19(\mathrm{t}, J=7.2 \mathrm{~Hz}, 3 \mathrm{H}), 3.17(\mathrm{q}, J=7.2 \mathrm{~Hz}, 2 \mathrm{H}), 6.64(\mathrm{~m}$, 2H), $6.73(\mathrm{~m}, 1 \mathrm{H}), 7.21(\mathrm{~m}, 2 \mathrm{H}) ;{ }^{13} \mathrm{C}$ NMR $\left(400 \mathrm{MHz}, \mathrm{CDCl}_{3}, \delta_{\mathrm{C}}\right)$ 15.1, 38.7, 113.0, 117.4, 129.5, 148.7; GCMS (EI) $m / z 121$. 
N-Ethyl-methylaniline: Starting from $p$-toluidine $\left(0.106 \mathrm{~g}, 9.9 \times 10^{-4} \mathrm{~mol}\right)$ using general procedure B gave a yellow-orange liquid $(0.119 \mathrm{~g}, 95 \%) . R_{\mathrm{f}} 0.43$ (10:1 hexanes:ethyl acetate); ${ }^{1} \mathrm{H}$ NMR $\left(400 \mathrm{MHz}, \mathrm{CDCl}_{3}, \delta_{\mathrm{H}}\right) 1.33(\mathrm{t}, J=7.2 \mathrm{~Hz}, 3 \mathrm{H}), 2.36(\mathrm{~s}, 3 \mathrm{H}), 3.22(\mathrm{q}, J$ $=7.2 \mathrm{~Hz}, 2 \mathrm{H}), 6.64(\mathrm{~d}, J=8.4 \mathrm{~Hz}, 2 \mathrm{H}), 7.10(\mathrm{~d}, J=8.4 \mathrm{~Hz}, 2 \mathrm{H}) ;{ }^{13} \mathrm{C} \mathrm{NMR}(400 \mathrm{MHz}$, $\left.\mathrm{CDCl}_{3}, \delta_{\mathrm{C}}\right) 15.3,20.7,39.1,113.3,126.7 ; 130.1,146.6$; GCMS (EI) $m / z 135$.

N-Ethyl-4-methoxyaniline: Starting from $p$-anisidine $\left(0.103 \mathrm{~g}, 8.43 \times 10^{-4} \mathrm{~mol}\right)$, acetonitrile $\left(4.0 \mathrm{~mL}, 7.3 \times 10^{-2} \mathrm{~mol}\right)$ using general procedure $\mathrm{D}$ gave an orange-brown liquid product $(0.126 \mathrm{~g}, 100 \%) . R_{\mathrm{f}} 0.41$ (10:1 hexanes:ethyl acetate); ${ }^{1} \mathrm{H}$ NMR (400 $\left.\mathrm{MHz}, \mathrm{CDCl}_{3}, \delta_{\mathrm{H}}\right) 1.26(\mathrm{t}, J=7.2 \mathrm{~Hz}, 3 \mathrm{H}), 3.13(\mathrm{q}, J=7.2 \mathrm{~Hz}, 2 \mathrm{H}), 3.77(\mathrm{~s}, 3 \mathrm{H}), 6.62$ $(\mathrm{d}, J=8.8 \mathrm{~Hz}, 2 \mathrm{H}), 6.82(\mathrm{~d}, J=9.2 \mathrm{~Hz}, 2 \mathrm{H}) ;{ }^{13} \mathrm{C} \mathrm{NMR}\left(400 \mathrm{MHz}, \mathrm{CDCl}_{3}, \delta_{\mathrm{C}}\right) 15.2,39.7$, 56.0, 114.4, 115.1, 143.0, 152.3; GCMS (EI) $m / z 151$.

N-Ethyl-2-methoxyaniline: Starting from 2-nitroanisole $\left(0.11 \mathrm{~g}, 7.18 \times 10^{-4} \mathrm{~mol}\right)$ and using general procedure B gave a yellow-orange liquid $(0.104 \mathrm{~g}, 96 \%) . R_{\mathrm{f}} 0.71(10: 1$ hexanes:ethyl acetate); ${ }^{1} \mathrm{H}$ NMR (400 MHz, $\left.\mathrm{CDCl}_{3}, \delta_{\mathrm{H}}\right) 1.33(\mathrm{t}, J=7.2 \mathrm{~Hz}, 3 \mathrm{H}), 3.20$ (q, $J=7.2 \mathrm{~Hz}, 2 \mathrm{H}), 3.88(\mathrm{~s}, 3 \mathrm{H}), 6.64-6.73(\mathrm{~m}, 2 \mathrm{H}), 6.79-6.82(\mathrm{~m}, 1 \mathrm{H}), 6.90-6.94(\mathrm{~m}, 1 \mathrm{H})$; ${ }^{13} \mathrm{C}$ NMR $\left(400 \mathrm{MHz}, \mathrm{CDCl}_{3}, \delta_{\mathrm{C}}\right) 15.1,38.4,55.6,109.6,110.1,116.5,121.6,138.7$, 147.0; GCMS (EI) $m / z 151$.

N-Ethyl-2-methoxyaniline: Starting from $o$-anisidine $\left(0.1 \mathrm{~g}, 8.1 \times 10^{-4} \mathrm{~mol}\right)$ and using general procedure B gave a yellow-orange liquid $(0.12 \mathrm{~g}, 98 \%) . R_{\mathrm{f}} 0.68(10: 1$ hexanes:ethyl acetate); ${ }^{1} \mathrm{H}$ NMR (400 MHz, $\left.\mathrm{CDCl}_{3}, \delta_{\mathrm{H}}\right) 1.35(\mathrm{t}, J=7.2 \mathrm{~Hz}, 3 \mathrm{H}), 3.23(\mathrm{~m}$, 
2H), $3.90(\mathrm{~s}, 3 \mathrm{H}), 4.18$ (br. s, $1 \mathrm{H})$, 6.67-6.75 (m, 2H), 6.82-6.84 (m, 1H), 6.93-6.97 (m, $1 \mathrm{H}) ;{ }^{13} \mathrm{C}$ NMR $\left(400 \mathrm{MHz}, \mathrm{CDCl}_{3}, \delta_{\mathrm{C}}\right) 15.2,38.4,55.61,109.6,110.1,116.5,121.6$, 138.7, 147.0; GCMS (EI) $m / z 151$.

N-Ethyl-2-methylaniline: Starting from 2-nitrotoluene $\left(0.10 \mathrm{~g}, 7.3 \times 10^{-4} \mathrm{~mol}\right)$ and using general procedure B gave a clear, colorless liquid $(0.081 \mathrm{~g}, 82.4 \%) . R_{\mathrm{f}} 0.71(10: 1$ hexanes:ethyl acetate); ${ }^{1} \mathrm{H}$ NMR (400 MHz, $\left.\mathrm{CDCl}_{3}, \delta_{\mathrm{H}}\right) 1.37$ (t, $\left.J=7.2 \mathrm{~Hz}, 3 \mathrm{H}\right), 2.20$ (s, $3 \mathrm{H}), 3.25(\mathrm{q}, J=7.2 \mathrm{~Hz}, 2 \mathrm{H}), \quad 6.67-6.74(\mathrm{~m}, 2 \mathrm{H}), 7.12(\mathrm{~d}, 1 \mathrm{H}), 7.18-7.22(\mathrm{~m}, 1 \mathrm{H}) ;{ }^{13} \mathrm{C}$ NMR (400 MHz, $\left.\mathrm{CDCl}_{3}, \delta_{\mathrm{C}}\right) 15.3,17.8,38.7,109.9,117.0 ; 122.0,127.4,130.29,146.7$; GCMS (EI) $m / z 135$.

N-Ethyl-methylaniline: Starting from $o$-toluidine $\left(0.10 \mathrm{~g}, 9.3 \times 10^{-4} \mathrm{~mol}\right)$ and using general procedure B gave a clear, colorless liquid product $(0.118 \mathrm{~g}, 94 \%): R_{\mathrm{f}} 0.32(10: 1$ hexanes:ethyl acetate); ${ }^{1} \mathrm{H} \mathrm{NMR}\left(400 \mathrm{MHz}, \mathrm{CDCl}_{3}, \delta_{\mathrm{H}}\right) 1.40(\mathrm{t}, J=7.2 \mathrm{~Hz}, 3 \mathrm{H}), 2.24$ (s, $3 \mathrm{H}), 3.29$ (q, $J=7.2 \mathrm{~Hz} 2 \mathrm{H}), \quad 6.71-6.78(\mathrm{~m}, 2 \mathrm{H}), 7.16(\mathrm{~d}, 1 \mathrm{H}), 7.22-7.26(\mathrm{~m}, 1 \mathrm{H}) ;{ }^{13} \mathrm{C}$ NMR (400 MHz, $\left.\mathrm{CDCl}_{3}, \delta_{\mathrm{C}}\right) 15.3,17.8,38.7,110.0,117.1 ; 122.0,127.5,130.3,146.7$; GCMS (EI) $m / z 135$.

N-Ethyl-3-methylaniline: Starting from 3-nitrotoluene $\left(0.10 \mathrm{~g}, 7.3 \times 10^{-4} \mathrm{~mol}\right)$ and using general procedure B gave a light-yellow liquid $(0.13 \mathrm{~g}, 100 \%): R_{\mathrm{f}} 0.71(10: 1$ hexanes:ethyl acetate); ${ }^{1} \mathrm{H} \mathrm{NMR}\left(400 \mathrm{MHz}, \mathrm{CDCl}_{3}, \delta_{\mathrm{H}}\right) 1.30(\mathrm{t}, J=7.2 \mathrm{~Hz}, 3 \mathrm{H}), 2.34$ (s, $3 \mathrm{H}), 3.19$ (q, $J=7.2 \mathrm{~Hz}, 2 \mathrm{H}), 3.53$ (br. s, $1 \mathrm{H}), 6.47-6.49$ (br. complex m, 2H), 6.59 (d, 
1H), 7.11-7.15 (m, 1H); ${ }^{13} \mathrm{C} \mathrm{NMR}\left(400 \mathrm{MHz}, \mathrm{CDCl}_{3}, \delta_{\mathrm{C}}\right)$ 15.2, 21.9, 38.8, 110.2, 117.02; 122.0, 127.4, 139.2, 148.8; GCMS (EI) $m / z 135$.

N-Ethyl-3-methylaniline: Starting from $m$-toluidine $\left(0.10 \mathrm{~g}, 9.3 \times 10^{-4} \mathrm{~mol}\right)$ and using general procedure $\mathrm{B}$ gave a yellow-orange liquid $(0.126 \mathrm{~g}, 100 \%): R_{\mathrm{f}} 0.70(10: 1$ hexanes:ethyl acetate); ${ }^{1} \mathrm{H}$ NMR (400 MHz, $\left.\mathrm{CDCl}_{3}, \delta_{\mathrm{H}}\right) 1.33(\mathrm{t}, J=7.2 \mathrm{~Hz}, 3 \mathrm{H}), 2.38$ (s, $3 \mathrm{H}), 3.22(\mathrm{q}, J=7.2 \mathrm{~Hz}, 2 \mathrm{H}), 6.51-6.52($ br. complex m, 2H), $6.63(\mathrm{~d}, 1 \mathrm{H}), 7.15-7.19(\mathrm{~m}$, $1 \mathrm{H}) ;{ }^{13} \mathrm{C}$ NMR $\left(400 \mathrm{MHz}, \mathrm{CDCl}_{3}, \delta_{\mathrm{C}}\right) 15.2,22.0,38.78,110.3,113.9 ; 118.5,129.4$, 139.2, 148.9; GCMS (EI) $m / z 135$.

N-Ethyl-3-methoxyaniline: Starting from $m$-anisidine $\left(0.10 \mathrm{~g}, 8.1 \times 10^{-4} \mathrm{~mol}\right)$ and using general procedure B gave an orange-brown liquid $(0.125 \mathrm{~g}, 86 \%): R_{\mathrm{f}} 0.47(10: 1$ hexanes:ethyl acetate); ${ }^{1} \mathrm{H}$ NMR (400 MHz, $\left.\mathrm{CDCl}_{3}, \delta_{\mathrm{H}}\right) 1.27(\mathrm{t}, J=7.2 \mathrm{~Hz}, 3 \mathrm{H}), 3.16$ (q, 2H), $3.80(\mathrm{~s}, 3 \mathrm{H}), 3.64$ (br. s, $1 \mathrm{H}), 6.20-6.21(\mathrm{~m}, 1 \mathrm{H}), 6.25-6.32(\mathrm{~m}, 2 \mathrm{H}), 7.10-7.14(\mathrm{~m}$, $1 \mathrm{H}) ;{ }^{13} \mathrm{C} \mathrm{NMR}\left(400 \mathrm{MHz}, \mathrm{CDCl}_{3}, \delta_{\mathrm{C}}\right) 15.1,38.7,55.3,109.6,102.5,106.2,130.2,150.2$, 161.12; GCMS (EI) $m / z 151$.

N-Ethyl hexylamine: : Starting from 1-nitrohexane $\left(0.10 \mathrm{~g}, 7.6 \times 10^{-4} \mathrm{~mol}\right)$ and using general procedure B gave a volatile product. GCMS (EI) $m / z 129$.

N-Ethyl cyclohexylamine: Starting from cyclohexylamine $\left(0.10 \mathrm{~g}, 1.0 \times 10^{-3} \mathrm{~mol}\right)$ and using general procedure B gave a light yellow liquid (0.05 g, $39 \%) .{ }^{1} \mathrm{H}$ NMR (400 MHz, $\left.\mathrm{CDCl}_{3}, \delta_{\mathrm{H}}\right)$ 1.23-1.27 (br. complex m, 3H), $1.48(\mathrm{t}, J=7.2 \mathrm{~Hz}, 3 \mathrm{H}$ ), 1.56-1.66 (br. 
complex m, 3H), 1.80-1.90 (br. complex m, 2H), 2.21-2.24 (br. complex m, 2H), 2.892.97 (br. complex m, $1 \mathrm{H}), 3.03(\mathrm{q}, J=7.2 \mathrm{~Hz}, 2 \mathrm{H}) ;{ }^{13} \mathrm{C} \mathrm{NMR}\left(400 \mathrm{MHz}, \mathrm{CDCl}_{3}, \delta_{\mathrm{C}}\right)$ 11.6, 24.8, 25.0, 29.3, 39.7, 56.9; GCMS (EI) $m / z 127$.

General Procedure C: Starting material (100 mg, nitroaryl or nitroalkyl) was dissolved in $\mathrm{MeOH}$ and stirred at room temperature. $5 \% \mathrm{Pd} / \mathrm{C}\left(0.039 \mathrm{~g}, 1.8 \times 10^{-5} \mathrm{~mol}\right)$ and $\mathrm{NH}_{4} \mathrm{HCO}_{2}\left(0.60 \mathrm{~g}, 9.5 \times 10^{-3} \mathrm{~mol}\right)$ dissolved in $\mathrm{H}_{2} \mathrm{O}(1.0 \mathrm{~mL})$ was then added to the reaction mixture while stirring. The reaction was monitored by TLC or GCMS (for nitroalkyl) for the in situ reduction of nitro compound to the corresponding primary amine. Nitrile ( 8 eq) was then added to the reaction mixture and stirring was continued until the primary amine intermediate (lowest $\mathrm{R}_{\mathrm{f}}$ ) was no longer detected by TLC. The reaction mixture was filtered, washed successively with methanol and $\mathrm{CH}_{2} \mathrm{Cl}_{2}$ and the filtrate evaporated in vacuo. The residue was dissolved in $\mathrm{CH}_{2} \mathrm{Cl}_{2}$ and the organic layer was separated, dried in $\mathrm{Na}_{2} \mathrm{SO}_{4}$ and filtered. The filtrate was then evaporated in vacuo to give the product.

N-Propyl-4-methoxyaniline: Starting from 4-nitroanisole $\left(0.05 \mathrm{~g}, 3.3 \times 10^{-4} \mathrm{~mol}\right)$, $\mathrm{MeOH}(2 \mathrm{~mL}), 5 \% \mathrm{Pd} / \mathrm{C}\left(0.0193 \mathrm{~g}, 9.1 \times 10^{-6} \mathrm{~mol}\right), \mathrm{NH}_{4} \mathrm{HCO}_{2}\left(0.30 \mathrm{~g}, 3.2 \times 10^{-3} \mathrm{~mol}\right)$, $\mathrm{H}_{2} \mathrm{O}(1.0 \mathrm{~mL})$, propionitrile $\left(0.186 \mathrm{~mL}, 2.66 \times 10^{-3} \mathrm{~mol}\right)$ using general procedure $\mathrm{C}$ gave a wine-red liquid (0.054 g, $99 \%$ ): $R_{\mathrm{f}} 0.27$ (10:1 hexanes:ethyl acetate); ${ }^{1} \mathrm{H}$ NMR (400 $\left.\mathrm{MHz}, \mathrm{CDCl}_{3}, \delta_{\mathrm{H}}\right) 1.01(\mathrm{t}, J=7.2 \mathrm{~Hz}, 3 \mathrm{H}), 1.60-1.68(\mathrm{~m}, 2 \mathrm{H}), 3.05$ (t, $\left.J=7.2 \mathrm{~Hz}, 2 \mathrm{H}\right)$, $3.76(\mathrm{~s}, 3 \mathrm{H}), 6.60(\mathrm{~d}, J=9.2 \mathrm{~Hz}, 2 \mathrm{H}), 6.80(\mathrm{~d}, J=8.8 \mathrm{~Hz}, 2 \mathrm{H}) ;{ }^{13} \mathrm{C} \mathrm{NMR}(400 \mathrm{MHz}$, $\left.\mathrm{CDCl}_{3}, \delta_{\mathrm{C}}\right) 11.9,23.1,47.1,56.1,114.3,115.13,143.1,152.2$; GCMS (EI) $m / z 165$. 
N-Butyl-4-methoxyaniline: Starting from 4-nitroanisole $\left(0.099 \mathrm{~g}, 6.5 \times 10^{-4} \mathrm{~mol}\right)$, $\mathrm{MeOH}(2 \mathrm{~mL}), 5 \% \mathrm{Pd} / \mathrm{C}\left(0.039 \mathrm{~g}, 1.86 \times 10^{-4} \mathrm{~mol}\right), \mathrm{NH}_{4} \mathrm{HCO}_{2}\left(0.499 \mathrm{~g}, 7.93 \times 10^{-3}\right.$ mol), $\mathrm{H}_{2} \mathrm{O}(1 \mathrm{~mL})$, butyronitrile $\left(4 \mathrm{~mL}, 4.57 \times 10^{-2} \mathrm{~mol}\right)$ using general procedure $\mathrm{C}$ gave an orange liquid (0.116 g, $100 \%): R_{\mathrm{f}} 0.34$ (10:1 hexanes:ethyl acetate); ${ }^{1} \mathrm{H}$ NMR (600 $\left.\mathrm{MHz}, \mathrm{CDCl}_{3}, \delta_{\mathrm{H}}\right) 0.95(\mathrm{t}, J=7.8 \mathrm{~Hz}, 3 \mathrm{H}), 1.39-1.45(\mathrm{~m}, 2 \mathrm{H}), 1.55-1.62(\mathrm{~m}, 2 \mathrm{H}), 3.06(\mathrm{t}$, $J=7.2 \mathrm{~Hz}, 2 \mathrm{H}), 3.74(\mathrm{~s}, 3 \mathrm{H}), 6.57(\mathrm{~d}, J=9.0 \mathrm{~Hz}, 2 \mathrm{H}), 6.80(\mathrm{~d}, J=9.0 \mathrm{~Hz}, 2 \mathrm{H}) ;{ }^{13} \mathrm{C}$ NMR $\left(400 \mathrm{MHz}, \mathrm{CDCl}_{3}, \delta_{\mathrm{C}}\right)$ 14.2, 20.1, 32.0, 44.9, 56.1, 114.2, 115.1, 143.1, 152.18; GCMS (EI) $m / z 179$.

N-Heptyl-4-methoxyaniline: Starting from 4-nitroanisole $\left(0.050 \mathrm{~g}, 3.25 \times 10^{-4} \mathrm{~mol}\right)$, $\mathrm{MeOH}(1.85 \mathrm{~mL}), 5 \% \mathrm{Pd} / \mathrm{C}\left(0.019 \mathrm{~g}, 8.9 \times 10^{-6} \mathrm{~mol}\right), \mathrm{NH}_{4} \mathrm{HCO}_{2}\left(0.299 \mathrm{~g}, 3.19 \times 10^{-3}\right.$ $\mathrm{mol}), \mathrm{H}_{2} \mathrm{O}(0.5 \mathrm{~mL})$, n-hexanoylnitrile $\left(0.16 \mathrm{~mL}, 1.0 \times 10^{-3} \mathrm{~mol}\right)$ using general procedure C gave a dark-brown oily liquid $(0.050 \mathrm{~g}, 74 \%): R_{\mathrm{f}} 0.41\left(10: 1\right.$ hexanes:ethyl acetate); ${ }^{1} \mathrm{H}$ NMR (600 MHz, $\left.\mathrm{CDCl}_{3}, \delta_{\mathrm{H}}\right) 0.90(\mathrm{t}, 3 \mathrm{H}), 1.29-1.44$ (br. complex m, 6H,), 1.56-1.64 (m, 2H), $3.06(\mathrm{t}, 2 \mathrm{H}), 3.74(\mathrm{~s}, 3 \mathrm{H}), 6.57(\mathrm{~d}, 2 \mathrm{H}), 6.78(\mathrm{~d}, 2 \mathrm{H}) ;{ }^{13} \mathrm{C} \mathrm{NMR}\left(400 \mathrm{MHz}, \mathrm{CDCl}_{3}\right.$, $\left.\delta_{\mathrm{C}}\right) 14.3,22.9,27.1,29.9,31.9,45.3,56.1,114.3,115.1,143.1,152.2 ;$ GCMS (EI) $m / z$ 207.

N-Propyl aniline: Starting from nitrobenzene $\left(0.1 \mathrm{~g}, 8.1 \times 10^{-4} \mathrm{~mol}\right), \mathrm{MeOH}(3.5 \mathrm{~mL})$, propionitrile $\left(0.59 \mathrm{~mL}, 6.74 \times 10^{-3} \mathrm{~mol}\right)$ using general procedure $\mathrm{C}$ gave a light brown liquid (0.095 g, $86 \%$ ). $R_{\mathrm{f}} 0.60$ (10:1 hexanes:ethyl acetate); ${ }^{1} \mathrm{H}$ NMR (400 MHz, $\mathrm{CDCl}_{3}$, $\left.\delta_{\mathrm{H}}\right) 1.04(\mathrm{t}, J=7.6 \mathrm{~Hz}, 3 \mathrm{H}), 1.63-1.72(\mathrm{~m}, 2 \mathrm{H}), 3.11(\mathrm{q}, J=6.8 \mathrm{~Hz}, 2 \mathrm{H}), 6.64(\mathrm{~m}, 2 \mathrm{H})$, 
$6.73(\mathrm{~m}, 1 \mathrm{H}), 7.21(\mathrm{~m}, 2 \mathrm{H}) ;{ }^{13} \mathrm{C} \mathrm{NMR}\left(400 \mathrm{MHz}, \mathrm{CDCl}_{3}, \delta_{\mathrm{C}}\right)$ 12.0, 23.0, 45.0, 113.0, 117.0, 129.5, 148.0; GCMS (EI) $m / z 135$.

N-Butyl aniline: Starting from nitrobenzene $\left(0.10 \mathrm{~g}, 8.1 \times 10^{-4} \mathrm{~mol}\right), \mathrm{MeOH}(3.4 \mathrm{~mL})$, butyronitrile $\left(0.57 \mathrm{~mL}, 6.6 \times 10^{-3} \mathrm{~mol}\right)$ using general procedure $\mathrm{C}$ gave a light brown liquid (0.085 g, $71 \%) . R_{\mathrm{f}} 0.58\left(10: 1\right.$ hexanes:ethyl acetate); ${ }^{1} \mathrm{H}$ NMR $\left(400 \mathrm{MHz}, \mathrm{CDCl}_{3}\right.$, $\left.\delta_{\mathrm{H}}\right) 0.99(\mathrm{t}, J=7.2 \mathrm{~Hz}, 3 \mathrm{H}), 1.41-1.50(\mathrm{~m}, 2 \mathrm{H}), 1.59-1.67(\mathrm{~m}, 2 \mathrm{H}), 3.13(\mathrm{q}, J=7.2 \mathrm{~Hz}$, $2 \mathrm{H}), 6.63(\mathrm{~m}, 2 \mathrm{H}), 6.71(\mathrm{~m}, 1 \mathrm{H}), 7.20(\mathrm{~m}, 2 \mathrm{H}) ;{ }^{13} \mathrm{C} \mathrm{NMR}\left(400 \mathrm{MHz}, \mathrm{CDCl}_{3}, \delta_{\mathrm{C}}\right)$ 14.1, 20.6, 32.0, 43.9, 113.0, 117.3, 129. 5, 148.8; GCMS (EI) $m / z 149$.

N-Propyl-4-methylaniline: Starting from 4-nitrotoluene (0.05 g, $\left.3.72 \times 10^{-4} \mathrm{~mol}\right)$, $\mathrm{MeOH}(1.8 \mathrm{~mL}), 5 \% \mathrm{Pd} / \mathrm{C}\left(0.019 \mathrm{~g}, 9.10 \times 10^{-6} \mathrm{~mol}\right), \mathrm{NH}_{4} \mathrm{HCO}_{2}\left(0.30 \mathrm{~g}, 3.2 \times 10^{-3} \mathrm{~mol}\right)$, propionitrile $\left(0.46 \mathrm{~mL}, 6.5 \times 10^{-3} \mathrm{~mol}\right)$ using general procedure $\mathrm{C}$ gave a light brown liquid $(0.055 \mathrm{~g}, 100 \%) . R_{\mathrm{f}} 0.48$ (10:1 hexanes:ethyl acetate); ${ }^{1} \mathrm{H}$ NMR $(400 \mathrm{MHz}$, $\left.\mathrm{CDCl}_{3}, \delta_{\mathrm{H}}\right) 1.02(\mathrm{t}, J=7.2 \mathrm{~Hz}, 3 \mathrm{H}), 1.62-1.71(\mathrm{~m}, 2 \mathrm{H}), 2.27(\mathrm{~s}, 3 \mathrm{H}), 3.09(\mathrm{t}, J=7.2 \mathrm{~Hz}$, $2 \mathrm{H}), 6.57(\mathrm{~d}, J=8.4 \mathrm{~Hz}, 2 \mathrm{H}), 7.02(\mathrm{~d}, J=8 \mathrm{~Hz}, 2 \mathrm{H}) ;{ }^{13} \mathrm{C} \mathrm{NMR}\left(400 \mathrm{MHz}, \mathrm{CDCl}_{3}, \delta_{\mathrm{C}}\right)$ 11.9, 20.6, 23.0, 46.5, 113.2, 126.5; 129.9, 146.6; GCMS (EI) $m / z 149$.

N-Butyl-4-methylaniline: Starting from 4-nitrotoluene (0.101 g, $\left.7.36 \times 10^{-4} \mathrm{~mol}\right)$, $\mathrm{MeOH}(3.5 \mathrm{~mL})$, butyronitrile $\left(0.510 \mathrm{~mL}, 2.92 \times 10^{-3} \mathrm{~mol}\right)$ using general procedure $\mathrm{C}$ gave a light brown liquid $(0.120 \mathrm{~g}, 100 \%) . R_{\mathrm{f}} 0.52$ (10:1 hexanes:ethyl acetate); ${ }^{1} \mathrm{H}$ NMR $\left(400 \mathrm{MHz}, \mathrm{CDCl}_{3}, \delta_{\mathrm{H}}\right) 0.98(\mathrm{t}, J=7.2 \mathrm{~Hz}, 3 \mathrm{H}), 1.41-1.50(\mathrm{~m}, 2 \mathrm{H}), 1.58-1.66(\mathrm{~m}, 2 \mathrm{H})$, $2.27(\mathrm{~s}, 3 \mathrm{H}), 3.11(\mathrm{t}, J=7.2 \mathrm{~Hz}, 2 \mathrm{H}), 6.56(\mathrm{~d}, J=8.4 \mathrm{~Hz}, 2 \mathrm{H}), 7.01(\mathrm{~d}, J=8 \mathrm{~Hz}, \mathrm{Ar}$, 
$2 \mathrm{H}) ;{ }^{13} \mathrm{C} \mathrm{NMR}\left(400 \mathrm{MHz}, \mathrm{CDCl}_{3}, \delta_{\mathrm{C}}\right) 14.1,20.6,20.6,32.0,44.3,113.2,126.5,129.9$, 146.6; GCMS (EI) $m / z 163$.

N-Propyl-2-methoxyaniline: Starting from 2-nitroanisole $\left(0.11 \mathrm{~g}, 7.2 \times 10^{-4} \mathrm{~mol}\right)$, $\mathrm{MeOH}(3.6 \mathrm{~mL})$, propionitrile $\left(0.41 \mathrm{~mL}, 5.75 \times 10^{-3} \mathrm{~mol}\right)$ using general procedure $\mathrm{C}$ gave a light yellow liquid $(0.119 \mathrm{~g}, 100 \%) . R_{\mathrm{f}} 0.69$ (10:1 hexanes:ethyl acetate); ${ }^{1} \mathrm{H}$ NMR $\left(400 \mathrm{MHz}, \mathrm{CDCl}_{3}, \delta_{\mathrm{H}}\right) 1.06(\mathrm{t}, J=7.2 \mathrm{~Hz}, 3 \mathrm{H}), 1.68-1.78(\mathrm{~m}, 2 \mathrm{H}), 3.14(\mathrm{~m}, 2 \mathrm{H}), 3.88(\mathrm{~s}$, $3 \mathrm{H},), \quad 4.25$ (br. s, $1 \mathrm{H}), 6.65-6.73(\mathrm{~m}, 2 \mathrm{H}), 6.80-6.82(\mathrm{~m}, 1 \mathrm{H}), 6.90-6.95(\mathrm{~m}, 1 \mathrm{H}) ;{ }^{13} \mathrm{C}$ NMR (400 MHz, $\left.\mathrm{CDCl}_{3}, \delta_{\mathrm{C}}\right)$ 12.0, 23.0, 45.8, 55.6, 109.6\, 110.0, 116.3, 121.6, 138.8, 147.0; GCMS (EI) $m / z 165$.

N-Butyl-2-methoxyaniline: Starting from 2-nitroanisole (0.11 g, $\left.7.2 \times 10^{-4} \mathrm{~mol}\right), \mathrm{MeOH}$ (3.5 mL), butyronitrile $\left(0.5 \mathrm{~mL}, 5.75 \times 10^{-3} \mathrm{~mol}\right)$ using general procedure $\mathrm{C}$ gave a yellow orange liquid product $(0.129 \mathrm{~g}, 100 \%) . R_{\mathrm{f}} 0.80$ (10:1 hexanes:ethyl acetate); ${ }^{1} \mathrm{H}$ NMR (400 MHz, $\left.\mathrm{CDCl}_{3}, \delta_{\mathrm{H}}\right) 0.98(\mathrm{t}, J=7.2 \mathrm{~Hz}, 3 \mathrm{H}), 1.28-1.38(\mathrm{~m}, 2 \mathrm{H}), 1.68-1.78(\mathrm{~m}$, $2 \mathrm{H}), 3.14(\mathrm{t}, J=7.2 \mathrm{~Hz}, 2 \mathrm{H}), 3.88$ (s, 3H), 4.20 (br. s, $1 \mathrm{H}), 6.61-6.69(\mathrm{~m}, 2 \mathrm{H}), 6.76-6.79$ (m, 1H), 6.87-6.91 (m, 1H); ${ }^{13} \mathrm{C}$ NMR (400 MHz, $\left.\mathrm{CDCl}_{3}, \delta_{\mathrm{C}}\right)$ 14.3, 21.0, 31.9, 43.6, 55.6, 109.5, 109.9, 116.3, 121.5, 138.8, 147.0; GCMS (EI) $m / z 179$.

N-Propyl-2-methylaniline: Starting from 2-nitrotoluene $\left(0.10 \mathrm{~g}, 7.3 \times 10^{-4} \mathrm{~mol}\right), \mathrm{MeOH}$ $(3.6 \mathrm{~mL})$, propionitrile $\left(0.42 \mathrm{~mL}, 5.83 \times 10^{-3} \mathrm{~mol}\right)$ using general procedure $\mathrm{C}$ gave a light yellow liquid (0.12 g, $100 \%): R_{\mathrm{f}} 0.76$ (10:1 hexanes:ethyl acetate); ${ }^{1} \mathrm{H}$ NMR $(400 \mathrm{MHz}$, $\left.\mathrm{CDCl}_{3}, \delta_{\mathrm{H}}\right) 1.06(\mathrm{t}, J=7.2 \mathrm{~Hz}, 3 \mathrm{H}), 1.68-1.77(\mathrm{~m}, 2 \mathrm{H}), 2.17(\mathrm{~s}, 3 \mathrm{H}), 3.16(\mathrm{t}, J=7.2 \mathrm{~Hz}$, 
2H), 6.64-6.70 (m, 2H), $7.08(\mathrm{~d}, 1 \mathrm{H}), 7.14-7.18(\mathrm{~m}, 1 \mathrm{H}) ;{ }^{13} \mathrm{C} \mathrm{NMR}\left(400 \mathrm{MHz}, \mathrm{CDCl}_{3}\right.$, $\left.\delta_{\mathrm{C}}\right) 12.0,17.7,23.0,46.0,109.8,116.9 ; 121.9,127.4,130.3,146.6$; GCMS (EI) $m / z 149$.

N-Butyl-2-methylaniline: Starting from 2-nitrotoluene (0.10 g, $\left.7.3 \times 10^{-4} \mathrm{~mol}\right), \mathrm{MeOH}$ ( $3.5 \mathrm{~mL})$, butyronitrile $\left(0.51 \mathrm{~mL}, 5.83 \times 10^{-3} \mathrm{~mol}\right)$ using general procedure $\mathrm{C}$ gave a light yellow liquid product $(0.127 \mathrm{~g}, 100 \%): R_{\mathrm{f}} 0.78$ (10:1 hexanes:ethyl acetate); ${ }^{1} \mathrm{H}$ NMR $\left(400 \mathrm{MHz}, \mathrm{CDCl}_{3}, \delta_{\mathrm{H}}\right) 1.00(\mathrm{t}, J=7.6 \mathrm{~Hz}, 3 \mathrm{H}), 1.43-1.53(\mathrm{~m}, 2 \mathrm{H}), 1.64-1.71(\mathrm{~m}, 2 \mathrm{H})$, $2.15(\mathrm{~s}, 3 \mathrm{H}), 3.17(\mathrm{t}, J=7.2 \mathrm{~Hz}, 2 \mathrm{H}), \quad 6.62-6.68(\mathrm{~m}, 2 \mathrm{H}), 7.07$ (d, 1H), 7.13-7.17 (m, $1 \mathrm{H}) ;{ }^{13} \mathrm{C} \mathrm{NMR}\left(400 \mathrm{MHz}, \mathrm{CDCl}_{3}, \delta_{\mathrm{C}}\right) 14.2,17.7,20.6,32.0,43.9,109.8,116.8 ; 121.9$, 127.4, 130.2, 146.7; GCMS (EI) $m / z 163$.

N-Propyl-3-methylaniline: Starting from 3-nitrotoluene ( $\left.0.1 \mathrm{~g}, 7.29 \times 10^{-4} \mathrm{~mol}\right), \mathrm{MeOH}$ (3.6 $\mathrm{mL})$, propionitrile $\left(0.42 \mathrm{~mL}, 5.83 \times 10^{-3} \mathrm{~mol}\right)$ using general procedure $\mathrm{C}$ gave a reddish-brown liquid (0.097 g, $89 \%$ ): $R_{\mathrm{f}} 0.59$ (10:1 hexanes:ethyl acetate); ${ }^{1} \mathrm{H}$ NMR (400 $\left.\mathrm{MHz}, \mathrm{CDCl}_{3}, \delta_{\mathrm{H}}\right) 1.08(\mathrm{t}, J=7.2 \mathrm{~Hz}, 3 \mathrm{H}), 1.68-1.73(\mathrm{~m}, 2 \mathrm{H}), 2.36(\mathrm{~s}, 3 \mathrm{H}), 3.14(\mathrm{t}, J=$ $7.2 \mathrm{~Hz}, 2 \mathrm{H}), 6.51$ (br. m, 2H), 6.59-6.61 (d, 1H), 7.12-7.16 (m, 1H); ${ }^{13} \mathrm{C}$ NMR $(400$ $\left.\mathrm{MHz}, \mathrm{CDCl}_{3}, \delta_{\mathrm{C}}\right) 12.0,21.9,23.1,46.1,110.2,113.8 ; 118.3,129.4,139.2,148.9 ; \mathrm{GCMS}$ (EI) $m / z 149$.

N-Butyl-3-methylaniline: Starting from 3-nitrotoluene (0.10 g, $\left.7.29 \times 10^{-4} \mathrm{~mol}\right), \mathrm{MeOH}$ (3.5 mL), butyronitrile $\left(0.51 \mathrm{~mL}, 5.83 \times 10^{-3} \mathrm{~mol}\right)$ using general procedure $\mathrm{C}$ gave a light yellow liquid $(0.119 \mathrm{~g}, 100 \%): R_{\mathrm{f}} 0.70$ (10:1 hexanes:ethyl acetate); ${ }^{1} \mathrm{H}$ NMR (400 MHz, $\left.\mathrm{CDCl}_{3}, \delta_{\mathrm{H}}\right) 0.97(\mathrm{t}, J=7.2 \mathrm{~Hz}, 3 \mathrm{H}), 1.39-1.49(\mathrm{~m}, 2 \mathrm{H}), 1.57-1.64(\mathrm{~m}, 2 \mathrm{H}), 2.29(\mathrm{~s}, 3 \mathrm{H})$, 
$3.10(\mathrm{t}, J=7.2 \mathrm{~Hz}, 2 \mathrm{H}), 6.42-6.44$ (br. complex m, 2H), $6.53(\mathrm{~d}, 1 \mathrm{H}), 7.05-7.09(\mathrm{~m}, 1 \mathrm{H})$

${ }^{13} \mathrm{C} \mathrm{NMR}\left(400 \mathrm{MHz}, \mathrm{CDCl}_{3}, \delta_{\mathrm{C}}\right)$ 14.2, 20.6, 21.9, 32.0, 43.9, 110.1, 113.69; 118.2, 129.3, 139.1, 148.9; GCMS (EI) $m / z 163$.

N-Propyl hexylamine: Starting from 1-nitrohexane (0.10 g, 7.6 x 10-4 mol), MeOH (3.6 $\mathrm{mL})$, propionitrile $\left(0.4 \mathrm{~mL}, 6.10 \times 10^{-3} \mathrm{~mol}\right)$ using general procedure $\mathrm{C}$ gave a light yellow liquid (0.044 g, $50 \%$ ): ${ }^{1} \mathrm{H}$ NMR $\left(400 \mathrm{MHz}, \mathrm{CDCl}_{3}, \delta_{\mathrm{H}}\right)$ 0.84-0.98 (complex m, 6H), 1.15-1.83 (br. complex m, 10H), 2.36 (m, 3H), 2.56 (m, 3H); ${ }^{13} \mathrm{C}$ NMR (400 MHz, $\left.\mathrm{CDCl}_{3}, \delta_{\mathrm{C}}\right) 11.8,14.4,22.9,23.2,28.1,30.7,32.6,49.9,52.2$; GCMS (EI) $m / z 143$.

General Procedure D: Starting material (100 mg, primary amine) was dissolved in $\mathrm{MeOH}$ and stirred at room temperature. $5 \% \mathrm{Pd} / \mathrm{C}\left(0.039 \mathrm{~g}, 1.8 \times 10^{-5} \mathrm{~mol}\right)$ and $\mathrm{NH}_{4} \mathrm{HCO}_{2}\left(0.60 \mathrm{~g}, 9.5 \times 10^{-3} \mathrm{~mol}\right)$ dissolved in $\mathrm{H}_{2} \mathrm{O}(1.0 \mathrm{~mL})$ was then added to the reaction mixture while stirring. Nitrile ( 8 eq) was then added to the reaction mixture and stirring was continued until the primary amine intermediate (lowest $\mathrm{R}_{\mathrm{f}}$ ) was no longer detected by TLC. The reaction mixture was filtered, washed successively with methanol and $\mathrm{CH}_{2} \mathrm{Cl}_{2}$ and the filtrate evaporated in vacuo. The residue was dissolved in $\mathrm{CH}_{2} \mathrm{Cl}_{2}$ and the organic layer was separated, dried in $\mathrm{Na}_{2} \mathrm{SO}_{4}$ and filtered. The filtrate was then evaporated in vacuo to give the product.

N-Ethyl aniline: Starting from aniline $\left(0.10 \mathrm{~g}, 1.1 \times 10^{-3} \mathrm{~mol}\right), \mathrm{MeOH}(3.3 \mathrm{~mL})$, acetonitrile $\left(0.45 \mathrm{~mL}, 8.56 \times 10^{-3} \mathrm{~mol}\right)$ using general procedure $\mathrm{D}$ gave a light yellow liquid $(0.108,83.0 \%) . R_{\mathrm{f}} 0.45$ (10:1 hexanes:ethyl acetate); ${ }^{1} \mathrm{H} \mathrm{NMR}\left(600 \mathrm{MHz}, \mathrm{CDCl}_{3}\right.$, 
$\left.\delta_{\mathrm{H}}\right) 1.20(\mathrm{t}, J=7.2 \mathrm{~Hz}, 3 \mathrm{H}), 3.19(\mathrm{q}, J=7.2 \mathrm{~Hz}, 2 \mathrm{H}), 6.65(\mathrm{~m}, 2 \mathrm{H}), 6.75(\mathrm{~m}, 1 \mathrm{H}), 7.22$ (m, 2H); ${ }^{13} \mathrm{C}$ NMR $\left(400 \mathrm{MHz}, \mathrm{CDCl}_{3}, \delta_{\mathrm{C}}\right) 15.3,38.8,113.1,117.6,129.6,148.8$; GCMS (EI) $m / z 121$.

N-Propyl-4-methoxyaniline: Starting from $p$-anisidine $\left(0.103 \mathrm{~g}, 8.42 \times 10^{-4} \mathrm{~mol}\right)$, MeOH (3.6 mL), propionitrile $\left(0.5 \mathrm{~mL}, 6.69 \times 10^{-3} \mathrm{~mol}\right)$ using general procedure $\mathrm{D}$ gave a yellow-orange liquid $(0.137 \mathrm{~g}, 99 \%) . R_{\mathrm{f}} 0.33$ (10:1 hexanes:ethyl acetate); ${ }^{1} \mathrm{H}$ NMR $\left(400 \mathrm{MHz}, \mathrm{CDCl}_{3}, \delta_{\mathrm{H}}\right) 1.01(\mathrm{t}, J=7.2 \mathrm{~Hz}, 3 \mathrm{H}), 1.62-1.67(\mathrm{~m}, 2 \mathrm{H}), 3.05(\mathrm{t}, J=7.2 \mathrm{~Hz}$, 2H), $3.76(\mathrm{~s}, 3 \mathrm{H}), 6.61(\mathrm{~d}, J=8.8 \mathrm{~Hz}, 2 \mathrm{H}), 6.81(\mathrm{~d}, J=8.8 \mathrm{~Hz}, 2 \mathrm{H}) ;{ }^{13} \mathrm{C}$ NMR $(400$ $\left.\mathrm{MHz}, \mathrm{CDCl}_{3}, \delta_{\mathrm{C}}\right) 11.9,23.1,47.1,56.1,114.3,115.1,143.1,152.1$; GCMS (EI) m/z 165.

N-Butyl-4-methoxyaniline: Starting from $p$-anisidine $\left(0.104 \mathrm{~g}, 8.42 \times 10^{-4} \mathrm{~mol}\right), \mathrm{MeOH}$ $(4.0 \mathrm{~mL})$, butyronitrile $\left(0.6 \mathrm{~mL}, 6.69 \times 10^{-3} \mathrm{~mol}\right)$ using general procedure $\mathrm{D}$ gave a yellow-orange liquid $(0.151 \mathrm{~g}, 100 \%) . \quad R_{\mathrm{f}} 0.37$ (10:1 hexanes:ethyl acetate); 0.99 (t, $J=$ $7.2 \mathrm{~Hz}, 3 \mathrm{H}), 1.43-1.48(\mathrm{~m}, 2 \mathrm{H}), 1.58-1.63(\mathrm{~m}, 2 \mathrm{H}), 3.09(\mathrm{t}, J=7.2 \mathrm{~Hz}, 2 \mathrm{H}), 3.77(\mathrm{~s}$, $3 \mathrm{H}), 6.61(\mathrm{~d}, J=8.8 \mathrm{~Hz}, 2 \mathrm{H}), 6.82(\mathrm{~d}, J=8.8 \mathrm{~Hz}, 2 \mathrm{H}) ;{ }^{13} \mathrm{C} \mathrm{NMR}\left(400 \mathrm{MHz}, \mathrm{CDCl}_{3}, \delta_{\mathrm{C}}\right)$ 14.2, 20.6, 32.0, 45.0, 56.0, 114.3, 115.1, 143.1, 152.2; GCMS (EI) $\mathrm{m} / z 179$.

N-Ethyl-aniline: Starting from aniline $\left(0.10 \mathrm{~g}, 1.07 \times 10^{-3} \mathrm{~mol}\right)$, MeOH $(3.6 \mathrm{~mL})$, acetonitrile $\left(0.45 \mathrm{~mL}, 8.56 \times 10^{-3} \mathrm{~mol}\right)$ using general procedure D gave a dark orangebrown liquid (0.08 g, $83 \%) . R_{\mathrm{f}} 0.59$ (10:1 hexanes:ethyl acetate); ${ }^{1} \mathrm{H}$ NMR (600 MHz, $\left.\mathrm{CDCl}_{3}, \delta_{\mathrm{H}}\right) 1.29(\mathrm{t}, J=7.2 \mathrm{~Hz}, 3 \mathrm{H}), 3.19(\mathrm{q}, J=7.2 \mathrm{~Hz}, 2 \mathrm{H}), 3.64$ (br. s, $\left.1 \mathrm{H}\right), 6.65(\mathrm{~m}$, 
2H), $6.72(\mathrm{~m}, 1 \mathrm{H}), 7.21(\mathrm{~m}, 2 \mathrm{H}) ;{ }^{13} \mathrm{C} \mathrm{NMR}\left(400 \mathrm{MHz}, \mathrm{CDCl}_{3}, \delta_{\mathrm{C}}\right)$ 15.2, 38.7, 113.0, 117.5, 129.5, 148.7; GCMS (EI) $m / z 121$.

N-Propyl aniline: Starting from aniline (0.1 g, $\left.1.07 \times 10^{-3} \mathrm{~mol}\right), \mathrm{MeOH}(3.3 \mathrm{~mL})$, propionitrile $\left(0.61 \mathrm{~mL}, 8.56 \times 10^{-3} \mathrm{~mol}\right)$ using general procedure $\mathrm{D}$ gave a light brown liquid product (0.09 g, 99.6\%). $R_{\mathrm{f}} 0.60$ (10:1 hexanes:ethyl acetate); ${ }^{1} \mathrm{H}$ NMR (400 MHz, $\left.\left.\mathrm{CDCl}_{3}, \delta_{\mathrm{H}}\right) 1.03(\mathrm{t}, J=7.6 \mathrm{~Hz}, 3 \mathrm{H}), 1.63-1.72(\mathrm{~m}, 2 \mathrm{H}), 3.11(\mathrm{q}, J=7.2 \mathrm{~Hz}, 2 \mathrm{H}),\right), 3.66$ (br. s, 1H), $6.64(\mathrm{~m}, 2 \mathrm{H}), 6.73(\mathrm{~m}, 1 \mathrm{H}), 7.21(\mathrm{~m}, 2 \mathrm{H},) ;{ }^{13} \mathrm{C} \mathrm{NMR}\left(400 \mathrm{MHz}, \mathrm{CDCl}_{3}, \delta_{\mathrm{C}}\right)$ 11.9, 23.0, 46.1, 113.0, 117.3, 129.5, 148.8; GCMS (EI) $m / z 135$.

N-Butyl aniline: Starting from aniline $\left(0.10 \mathrm{~g}, 1.07 \times 10^{-3} \mathrm{~mol}\right)$, $\mathrm{MeOH}(2.6 \mathrm{~mL})$, butyronitrile $\left(0.75 \mathrm{~mL}, 8.56 \times 10^{-3} \mathrm{~mol}\right)$ using general procedure $\mathrm{D}$ gave a brown liquid $(0.160 \mathrm{~g}, 100 \%) . R_{\mathrm{f}} 0.66\left(10: 1\right.$ hexanes:ethyl acetate); ${ }^{1} \mathrm{H}$ NMR $\left(400 \mathrm{MHz}, \mathrm{CDCl}_{3}, \delta_{\mathrm{H}}\right)$ $1.01(\mathrm{t}, J=7.2 \mathrm{~Hz}, 3 \mathrm{H}), 1.43-1.52(\mathrm{~m}, 2 \mathrm{H}), 1.60-1.68(\mathrm{~m}, 2 \mathrm{H}), 3.14(\mathrm{q}, J=7.2 \mathrm{~Hz}, 2 \mathrm{H})$, $6.65(\mathrm{~m}, 2 \mathrm{H}), 6.74(\mathrm{~m}, 1 \mathrm{H}), 7.22(\mathrm{~m}, 2 \mathrm{H}) ;{ }^{13} \mathrm{C} \mathrm{NMR}\left(400 \mathrm{MHz}, \mathrm{CDCl}_{3}, \delta_{\mathrm{C}}\right)$ 14.2, 20.6, 32.0, 44.0, 113.0, 117.3, 129.5, 148.9; GCMS (EI) $m / z 149$.

N-Propyl-4-methylaniline: Starting from $p$-toluidine $\left(0.103 \mathrm{~g}, 9.6 \times 10^{-4} \mathrm{~mol}\right), \mathrm{MeOH}$ $(3.5 \mathrm{~mL})$, , propionitrile $\left(0.46 \mathrm{~mL}, 6.5 \times 10^{-3} \mathrm{~mol}\right)$ using general procedure $\mathrm{D}$ gave a redorange liquid (0.143 g, $100 \%): R_{\mathrm{f}} 0.49$ (10:1 hexanes:ethyl acetate); ${ }^{1} \mathrm{H}$ NMR (400 MHz, $\left.\mathrm{CDCl}_{3}, \delta_{\mathrm{H}}\right) 1.01(\mathrm{t}, J=7.2 \mathrm{~Hz}, 3 \mathrm{H}), 1.62-1.70(\mathrm{~m}, 2 \mathrm{H}), 2.27(\mathrm{~s}, 3 \mathrm{H}), 3.07(\mathrm{t}, J=7.2 \mathrm{~Hz}$, $2 \mathrm{H}), 6.56(\mathrm{~d}, J=8.4 \mathrm{~Hz}, 2 \mathrm{H}), 7.01(\mathrm{~d}, J=8 \mathrm{~Hz}, 2 \mathrm{H}) ;{ }^{13} \mathrm{C} \mathrm{NMR}\left(400 \mathrm{MHz}, \mathrm{CDCl}_{3}, \delta_{\mathrm{C}}\right)$ 11.9, 20.6, 23.0, 46.5, 113.2, 126.5; 129.9, 146.5; GCMS (EI) $m / z 149$. 
N-Butyl-4-methylaniline: Starting from $p$-toluidine $\left(0.107 \mathrm{~g}, 9.96 \times 10^{-4} \mathrm{~mol}\right), \mathrm{MeOH}$ (3.3 mL), butyronitrile $\left(0.65 \mathrm{~mL}, 7.5 \times 10^{-3} \mathrm{~mol}\right)$ using general procedure $\mathrm{D}$ gave a redorange liquid $(0.163 \mathrm{~g}, 100 \%) . R_{\mathrm{f}} 0.50$ (10:1 hexanes:ethyl acetate); ${ }^{1} \mathrm{H}$ NMR (400 MHz, $\left.\mathrm{CDCl}_{3}, \delta_{\mathrm{H}}\right) 1.02(\mathrm{t}, J=7.6 \mathrm{~Hz}, 3 \mathrm{H}), 1.43-1.53(\mathrm{~m}, 2 \mathrm{H}), 1.60-1.68(\mathrm{~m}, 2 \mathrm{H}), 2.30(\mathrm{~s}, 3 \mathrm{H})$, $3.11(\mathrm{t}, J=7.2 \mathrm{~Hz}, 2 \mathrm{H}), 6.59(\mathrm{~d}, J=8.4 \mathrm{~Hz}, 2 \mathrm{H}), 7.04(\mathrm{~d}, J=8 \mathrm{~Hz}, 2 \mathrm{H}) ;{ }^{13} \mathrm{C} \mathrm{NMR}(400$ $\left.\mathrm{MHz}, \mathrm{CDCl}_{3}, \delta_{\mathrm{C}}\right) 14.2,20.6,20.7,32.0,44.3,113.2,126.4,130.0,146.6$; GCMS (EI) $m / z$ 163.

N-Propyl-2-methoxyaniline: Starting from o-anisidine $\left(0.10 \mathrm{~g}, 8.12 \times 10^{-4} \mathrm{~mol}\right), \mathrm{MeOH}$ (3.5 $\mathrm{mL})$, propionitrile $\left(0.46 \mathrm{~mL}, 6.5 \times 10^{-3} \mathrm{~mol}\right)$ using general procedure $\mathrm{D}$ gave a light yellow liquid $(0.134 \mathrm{~g}, 100 \%) . R_{\mathrm{f}} 0.62$ (10:1 hexanes:ethyl acetate); ${ }^{1} \mathrm{H}$ NMR $(400 \mathrm{MHz}$, $\left.\mathrm{CDCl}_{3}, \delta_{\mathrm{H}}\right) 1.07(\mathrm{t}, J=7.6 \mathrm{~Hz}, 3 \mathrm{H}), 1.70-1.78(\mathrm{~m}, 2 \mathrm{H}), 3.15(\mathrm{~m}, 2 \mathrm{H}), 3.89(\mathrm{~s}, 3 \mathrm{H}), 6.66-$ $6.74(\mathrm{~m}, 2 \mathrm{H}), 6.81-6.83(\mathrm{~m}, 1 \mathrm{H}), 6.92-6.96(\mathrm{~m}, 1 \mathrm{H}) ;{ }^{13} \mathrm{C} \mathrm{NMR}\left(400 \mathrm{MHz}, \mathrm{CDCl}_{3}, \delta_{\mathrm{C}}\right)$ 12.0, 23.0, 45.8, 55.6, 109.7, 110.0, 116.3, 121.6, 138.8, 147.0; GCMS (EI) m/z 165.

N-Butyl-2-methoxyaniline: Starting from o-anisidine $\left(0.10 \mathrm{~g}, 8.1 \mathrm{x} 10^{-4} \mathrm{~mol}\right), \mathrm{MeOH}$ (3.4 mL), butyronitrile $\left(0.57 \mathrm{~mL}, 6.50 \times 10^{-3} \mathrm{~mol}\right)$ using general procedure $\mathrm{D}$ gave a yellow orange liquid $(0.150 \mathrm{~g}, 100 \%) . R_{\mathrm{f}} 0.78\left(10: 1\right.$ hexanes:ethyl acetate); ${ }^{1} \mathrm{H}$ NMR (400 MHz, $\left.\mathrm{CDCl}_{3}, \delta_{\mathrm{H}}\right) 1.02(\mathrm{t}, J=7.6 \mathrm{~Hz}, 3 \mathrm{H}), 1.44-1.54(\mathrm{~m}, 2 \mathrm{H}), 1.66-1.73(\mathrm{~m}, 2 \mathrm{H})$, $3.17(\mathrm{~m}, 2 \mathrm{H}), 3.88(\mathrm{~s}, 3 \mathrm{H}), 4.22$ (br. s, 1H), 6.65-6.72 (m, 2H), 6.80-6.82 (m, 1H), 6.90$6.95(\mathrm{~m}, 1 \mathrm{H}) ;{ }^{13} \mathrm{C} \mathrm{NMR}\left(400 \mathrm{MHz}, \mathrm{CDCl}_{3}, \delta_{\mathrm{C}}\right) 14.2,20.7,31.9,43.7,55.6,109.6,110.0$, 116.3, 121.6, 138.8, 147.0; GCMS (EI) $m / z 179$. 
N-Propyl-2-methylaniline: Starting from $o$-toluidine $\left(0.100 \mathrm{~g}, 9.3 \times 10^{-4} \mathrm{~mol}\right), \mathrm{MeOH}$ $(3.5 \mathrm{~mL})$, propionitrile $\left(0.53 \mathrm{~mL}, 7.4 \times 10^{-3} \mathrm{~mol}\right)$ using general procedure $\mathrm{D}$ gave a light yellow liquid (0.139 g, $99.9 \%$ ): $R_{\mathrm{f}} 0.77$ (10:1 hexanes:ethyl acetate); ${ }^{1} \mathrm{H}$ NMR (400 $\left.\mathrm{MHz}, \mathrm{CDCl}_{3}, \delta_{\mathrm{H}}\right) 1.14(\mathrm{t}, J=7.2 \mathrm{~Hz}, 3 \mathrm{H}), 1.75-1.85(\mathrm{~m}, 2 \mathrm{H}), 2.24(\mathrm{~s}, 3 \mathrm{H}), 3.23(\mathrm{t}, J=$ $7.2 \mathrm{~Hz}, 2 \mathrm{H}), 3.54$ (br. s, $1 \mathrm{H}), 6.72-6.78(\mathrm{~m}, 2 \mathrm{H}), 7.16(\mathrm{~d}, 1 \mathrm{H}), 7.22-7.27(\mathrm{~m}, 1 \mathrm{H}) ;{ }^{13} \mathrm{C}$ NMR $\left(400 \mathrm{MHz}, \mathrm{CDCl}_{3}, \delta_{\mathrm{C}}\right) 12.0,17.8,23.1,46.1,109.9,116.9 ; 122.0,127.5,130.4$, 146.7; GCMS (EI) $m / z 149$.

N-Butyl-2-methylaniline: Starting from $o$-toluidine $\left(0.100 \mathrm{~g}, 9.3 \times 10^{-4} \mathrm{~mol}\right) \mathrm{MeOH}(3.3$ $\mathrm{mL})$, butyronitrile $\left(0.65 \mathrm{~mL}, 7.5 \times 10^{-3} \mathrm{~mol}\right)$ using general procedure $\mathrm{D}$ gave a light yellow liquid (0.152 g, $100 \%): R_{\mathrm{f}} 0.76$ (10:1 hexanes:ethyl acetate); ${ }^{1} \mathrm{H}$ NMR (400 MHz, $\left.\mathrm{CDCl}_{3}, \delta_{\mathrm{H}}\right) 1.01(\mathrm{t}, J=7.6 \mathrm{~Hz}, 3 \mathrm{H}), 1.44-1.54(\mathrm{~m}, 2 \mathrm{H}), 1.64-1.71(\mathrm{~m}, 2 \mathrm{H}), 2.16(\mathrm{~s}, 3 \mathrm{H})$, $3.18(\mathrm{t}, J=7.2 \mathrm{~Hz}, 2 \mathrm{H}), 6.63-6.69(\mathrm{~m}, 2 \mathrm{H}), 7.08(\mathrm{~d}, 1 \mathrm{H}), 7.14-7.18(\mathrm{~m}, 1 \mathrm{H}) ;{ }^{13} \mathrm{C} \mathrm{NMR}$ $\left(400 \mathrm{MHz}, \mathrm{CDCl}_{3}, \delta_{\mathrm{C}}\right) 14.2,17.7,20.7,32.0,43.9,109.8,116.8,121.9,127.4,130.2$, 146.7; GCMS (EI) $m / z 163$.

N-Propyl-3-methylaniline: Starting from $m$-toluidine $\left(0.10 \mathrm{~g}, 9.3 \times 10^{-4} \mathrm{~mol}\right), \mathrm{MeOH}$ (3.5 $\mathrm{mL}$,) propionitrile $\left(0.52 \mathrm{~mL}, 7.46 \times 10^{-3} \mathrm{~mol}\right)$, reddish-brown liquid $(0.132 \mathrm{~g}, 100$ \%): $R_{\mathrm{f}} 0.59$ (10:1 hexanes:ethyl acetate); ${ }^{1} \mathrm{H}$ NMR $\left(400 \mathrm{MHz}, \mathrm{CDCl}_{3}, \delta_{\mathrm{H}}\right) 1.12(\mathrm{t}, J=7.2$ Hz, 3H), 1.70-1.79 (m, 2H), 2.41 (s, 3H), 3.17 (t, $J=7.2 \mathrm{~Hz}, 2 \mathrm{H}), 6.55$ (br. m, 2H), 6.64-6.66 (d, 1H), 7.17-7.21 (m, 1H); ${ }^{13} \mathrm{C}$ NMR $\left(400 \mathrm{MHz}, \mathrm{CDCl}_{3}, \delta_{\mathrm{C}}\right)$ 12.0, 22.0, 23.1, 46.2, 110.2, 113.8; 118.3, 129.4, 139.2, 149.0; GCMS (EI) $m / z 149$. 
N-Butyl-3-methylaniline: Starting from $m$-toluidine $\left(0.10 \mathrm{~g}, 9.3 \times 10^{-4} \mathrm{~mol}\right), \mathrm{MeOH}(3.3$ $\mathrm{mL})$, butyronitrile $\left(0.65 \mathrm{~mL}, 7.5 \times 10^{-3} \mathrm{~mol}\right)$ using general procedure $\mathrm{D}$ gave a light yellow liquid product $(0.127 \mathrm{~g}, 100 \%) . R_{\mathrm{f}} 0.70\left(10: 1\right.$ hexanes:ethyl acetate); ${ }^{1} \mathrm{H}$ NMR (400 MHz, $\left.\mathrm{CDCl}_{3}, \delta_{\mathrm{H}}\right) 1.00(\mathrm{t}, J=7.2 \mathrm{~Hz}, 3 \mathrm{H}), 1.42-1.49(\mathrm{~m}, 2 \mathrm{H}), 1.59-1.65(\mathrm{~m}, 2 \mathrm{HH})$, 2.32 (s, 3H), 3.13 (t, $J=7.2 \mathrm{~Hz}, 2 \mathrm{H}$ ), 3.57 (br. s, 1H), 6.45-6.46 (br. complex m, 2H), $6.55(\mathrm{~d}, 1 \mathrm{H}), 7.08-7.12(\mathrm{~m}, 1 \mathrm{H}) ;{ }^{13} \mathrm{C} \mathrm{NMR}\left(400 \mathrm{MHz}, \mathrm{CDCl}_{3}, \delta_{\mathrm{C}}\right)$ 14.2, 20.6, 21.9, 32.0, 44.0, 110.1, 113.7, 118.3, 129.3, 139.2, 148.9; GCMS (EI) $m / z 163$.

N-Propyl-3-methoxyaniline: Starting from $m$-anisidine $\left(0.10 \mathrm{~g}, 8.12 \times 10^{-4} \mathrm{~mol}\right), \mathrm{MeOH}$ $(3.5 \mathrm{~mL})$, propionitrile $\left(0.46 \mathrm{~mL}, 6.50 \times 10^{-3} \mathrm{~mol}\right)$ using general procedure $\mathrm{D}$ gave a brown liquid (0.134 g, $100 \%): R_{\mathrm{f}} 0.44$ (10:1 hexanes:ethyl acetate); ${ }^{1} \mathrm{H}$ NMR (400 MHz, $\left.\mathrm{CDCl}_{3}, \delta_{\mathrm{H}}\right) 1.02(\mathrm{t}, J=7.2 \mathrm{~Hz}, 3 \mathrm{H}), 1.61-1.70(\mathrm{~m}, 2 \mathrm{H}), 3.009(\mathrm{t}, J=7.2 \mathrm{~Hz}, 2 \mathrm{H}), 3.80(\mathrm{~s}$, $3 \mathrm{H}), \quad 6.19-6.20(\mathrm{~m}, 1 \mathrm{H}), 6.24-6.30(\mathrm{~m}, 2 \mathrm{H}), 7.08-7.13(\mathrm{~m}, 1 \mathrm{H}) ;{ }^{13} \mathrm{C}$ NMR $(400 \mathrm{MHz}$, $\left.\mathrm{CDCl}_{3}, \delta_{\mathrm{C}}\right) 11.9,23.0,46.0,55.3,98.9,102.4,106.2,130.2,150.2,161.2 ;$ GCMS (EI) $m / z 165$.

N-Butyl-3-methoxyaniline: Starting from $m$-anisidine $\left(0.10 \mathrm{~g}, 8.1 \times 10^{-4} \mathrm{~mol}\right), \mathrm{MeOH}$ (3.43 mL), butyronitrile $\left(0.57 \mathrm{~mL}, 6.50 \times 10^{-3} \mathrm{~mol}\right)$ using general procedure $\mathrm{D}$ gave an orange brown liquid product $(0.146 \mathrm{~g}, 100 \%): R_{\mathrm{f}} 0.72\left(10: 1\right.$ hexanes:ethyl acetate); ${ }^{1} \mathrm{H}$ NMR (400 MHz, $\left.\mathrm{CDCl}_{3}, \delta_{\mathrm{H}}\right) 0.93(\mathrm{t}, J=7.2 \mathrm{~Hz}, 3 \mathrm{H}), 1.39-1.47(\mathrm{~m}, 2 \mathrm{H}), 1.55-1.63(\mathrm{~m}$, 2H), 3.06-3.11 (m, 2H), $3.76(\mathrm{~s}, 3 \mathrm{H}), 6.16-6.17(\mathrm{~m}, 1 \mathrm{H}), 6.21-6.27(\mathrm{~m}, 2 \mathrm{H})$, 7.05-7.09 
(m, 1H); ${ }^{13} \mathrm{C} \mathrm{NMR}\left(400 \mathrm{MHz}, \mathrm{CDCl}_{3}, \delta_{\mathrm{C}}\right)$ 14.4, 31.9, 43.9, 54.2, 55.2, 98.8, 102.3, 106.1, 130.1, 150.2, 161.1; GCMS (EI) $m / z 179$.

N-Propyl cyclohexylamine: Starting from cyclohexylamine $\left(0.10 \mathrm{~g}, 1.0 \times 10^{-3} \mathrm{~mol}\right)$, propionitrile $\left(0.58 \mathrm{~mL}, 8.08 \times 10^{-3} \mathrm{~mol}\right)$ using general procedure D gave a light yellow liquid product (0.102 g, $77 \%$ ). ${ }^{1} \mathrm{H} \mathrm{NMR}\left(400 \mathrm{MHz}, \mathrm{CDCl}_{3}, \delta_{\mathrm{H}}\right)$ 0.76-0.80 (complex m, $1 \mathrm{H}), 0.84(\mathrm{t}, J=7.2 \mathrm{~Hz}, 3 \mathrm{H}),, 0.94-1.04(\mathrm{brm}, 2 \mathrm{H}), 1.05-1.22$ (brm, 3H), 1.37-1.46 (m, 2H), 1.60-1.70 (brm, 2H), 1.77-1.82 (brm, 2H), 2.27-2.37 (brm, 1H), 2.51 (t, $J=7.2 \mathrm{~Hz}$, $3 \mathrm{H}) ;{ }^{13} \mathrm{C}$ NMR (400 MHz, $\left.\mathrm{CDCl}_{3}, \delta_{\mathrm{C}}\right)$ 12.0, 23.8, 25.3, 26.4, 33.9, 49.1, 57.0; GCMS (EI) $m / z 141$.

N-butyl cyclohexylamine: Starting from cyclohexylamine $\left(0.25 \mathrm{~g}, 2.5 \times 10^{-3} \mathrm{~mol}\right)$, butyronitrile $\left(1.77 \mathrm{~mL}, 2.02 \times 10^{-2} \mathrm{~mol}\right)$ using general procedure $\mathrm{D}$ gave an oily crystalline solid $(0.334 \mathrm{~g}, 85 \%) .{ }^{1} \mathrm{H}$ NMR $\left(400 \mathrm{MHz}, \mathrm{CDCl}_{3}, \delta_{\mathrm{H}}\right) 0.82(\mathrm{t}, J=7.2 \mathrm{~Hz}$, 3H), 1.00-1.94 (brm, 14H), 2.64 (complex m, 1H); $\left.{ }^{13} \mathrm{C} \mathrm{NMR} \mathrm{(400} \mathrm{MHz,} \mathrm{CDCl}_{3}, \delta_{\mathrm{C}}\right)$ 14.3, 21.0, 25.3, 29.3, 33.3, 46.6, 54.0, 57.1; GCMS (EI) $m / z 155$. 